\title{
An Inverse Capacitated Transportation Problem
}

\author{
${ }^{1}$ Sanjay Jain, ${ }^{2}$ Nitin Arya \\ Department of Mathematical Sciences, Government College, Ajmer \\ Affiliated to M. D. S. University, Ajmer - 305 001, INDIA \\ Department of Mathematics, Government Engineering College, Jhalawar \\ Affiliated to Rajasthan Technical University, Kota, INDIA
}

\begin{abstract}
This paper deal with the inverse version of capacitated transportation problem, in which the unit transportation cost of some cells in the original problem are adjusted as little as possible so that the given feasible solution become an optimal one. In our proposed method, first we reduce the capacitated transportation problem into linear programming problem and then obtained its inverse problem using dual and the optimality conditions. The method has been illustrated by a numerical example also.
\end{abstract}

Keywords: Inverse optimization, Capacitated transportation problem, Complementary slackness, Optimal Solution

\section{Introduction}

Inverse optimization is a relatively new area of research and study of inverse optimization is useful in many branches. Since last 20 years, many applications of inverse optimization have been found in different areas such as: geophysical sciences, traffic equilibrium, isotonic regression, portfolio optimization etc. Generally, in an optimization problem, it is assumed that all the parameter associated with the objective function and constraints are known and solve the problem for a solution which is optimal for the given parameter values. In practice, there are many situations where the parameter values are not known with certain, but some estimates of these parameters are given and also from the past experience or past practice, we have an optimal solution. In such situations, inverse optimization can be used to adjust the parameter values as little as possible so that the given feasible solution becomes optimal.

Burton and Toint [1] were the first who investigate the inverse optimization for shortest path problem under $l_{2}$ norm, since then a lot of work has been done on inverse optimization but most of the work is based on combinatorial optimization problems. Zhang and Liu [2] have first been calculated some inverse linear programming problem and further investigated inverse linear programming problems in [3]. Ahuja and Orlin [4] provide various references in the area of inverse optimization and compile several applications in network flow problems with unit weight and develop combinatorial proofs of correctness. Huang and Liu [5] and Amin and Emrouznejad [6], have considered applications of inverse problem. Yibing, Tiesong and Zhongping [7] worked on inverse optimal value problem Zhang and Zhang [8-10] worked on inverse quadratic programming problems, and Wang [11] has given the cutting plane algorithm for inverse integer programming problem. Milan Hladik [12] have first been considered inverse problem for generalized linear fractional programming and Jaing, Xiao, Zhang and Zhang [13] worked on inverse linear programming. Jain, Arya [14] have given an inverse model for linear fractional programming problems.

A transportation problem is a minimization problem of the cost of transportation from some origins to some other destinations. The minimum cost planning plays an important role for solving the transportation problem from origins to different destinations, such as from factories to warehouses or from warehouses to supermarkets, etc. A transportation problem in which total availability of origins and total requirement of destinations is not met then it is known as unbalanced transportation problem.

A capacitated transportation problem seeks to optimize a linear objective function subject to linear constraints and non negative, upper bound restrictions on the variables. Hitchcock [15] was the first, who formulated and solved the classical transportation problem, Kantorivich [16] worked on the translocation of masses, Kantorivich, and Gavurin [17] authored an application base study on capacitated transportation problem. Dantzig and Thapa [18] compile the literature on linear programming problems, Jain[19] have given the dual matrix approach for unbalanced transportation, and a lot of work have been done by numerous researchers on different types of transportation problems.

This paper is organized as follows. In section 2 the problem and its mathematical formulation are presented. In section 3 the method is stepwise stated. In section 4 an example is given to illustrated our proposed method. Finally conclusion is given in the last section. 


\section{The Problem}

In this paper, we have discussed an inverse optimization method for capacitated transportation problem. Ahuja, Orlin [4] play the key role in our discussion, they have given the inverse version of linear programming problem and formulate it as a linear programming problem. In the similar way, we have considered the following linear programming formulation of the capacitated transportation problem

$$
\begin{array}{lll}
\operatorname{Min} f(\mathrm{x})= & \sum_{i=1}^{m} \sum_{j=1}^{n} c_{i j} x_{i j} & \\
\text { s.t. } & \sum_{j=1}^{n} x_{i j}=a_{i}, & i=1,2, \ldots, m, \\
& \sum_{i=1}^{m} x_{i j}=b_{j}, & j=1,2, \ldots, n, \\
& 0 \leq x_{i j} \leq h_{i j}, i=1,2, \ldots, m, j=1,2, \ldots, n .
\end{array}
$$

If $\mathrm{x}_{0}$ is the given feasible solution then the inverse problem under $l_{1}$ norm is the following linear program:

Min $\quad \sum_{i} \sum_{j}\left(\alpha_{i j}+\beta_{i j}\right)$

s.t. $\quad u_{i}+v_{j}+\lambda_{i j}-\alpha_{i j}+\beta_{i j}=c_{i j} \quad$ for all $(i, j) \in L$

$$
\begin{aligned}
& u_{i}+v_{j}-\phi_{i j}-\alpha_{i j}+\beta_{i j}=c_{i j} \quad \text { for all }(i, j) \in U \\
& u_{i}+v_{j} \quad-\alpha_{i j}+\beta_{i j}=c_{i j} \quad \text { for all }(i, j) \in F \\
& \alpha_{i j}, \beta_{i j}, \lambda_{i j}, \phi_{i j} \geq 0, i=1,2, \ldots, m, \quad j=1,2, \ldots, n .
\end{aligned}
$$

Where $L, F, U$ are index sets defined as $L=\left\{(i, j) / x_{i j}^{0}=0\right\}, F=\left\{(i, j) / 0<x_{i j}^{0}<h_{i j}\right\}$ and $U=$ $\left\{(i, j) / x_{i j}^{0}=h_{i j}\right\}$.

\section{The method}

In our proposed method, we have considered the linear programming formulation of the capacitated transportation problem and obtained its inverse problem as a linear program using its dual and optimality conditions. Then we obtained the modified cost coefficients using the optimality conditions for the transportation problem. At the and of this discussion we have shown through an example that the optimal dual variables calculated in the final transportation table of two phase method can be used to obtain the modified cost and alternate optimal solution.

\section{Inverse capacitated transportation problem}

Dual of the LPP (1) is the following linear program:

$$
\begin{array}{ll}
\text { Max } & \sum_{i=1}^{m} a_{i} u_{i}+\sum_{j=1}^{n} b_{j} v_{j}-\sum_{i=1}^{m} \sum_{j=1}^{n} h_{i j} \phi_{i j} \\
\text { s.t. } & u_{i}+v_{j}+\lambda_{i j}-\phi_{i j}=c_{i j}, i=1,2, \ldots, m, j=1,2, \ldots, n, \\
& \lambda_{i j} \geq 0, \phi_{i j} \geq 0, i=1,2, \ldots, m, j=1,2, \ldots, n .
\end{array}
$$

Where $u_{i}, v_{j}, \lambda_{i j}$ and $\phi_{i j}$ are the dual variables.

One form of the optimality condition for linear programming states that the primal solution $x$ and dual solution $(u, v, \lambda, \phi)$ are optimal for respective problems if both $x$ and $(u, v, \lambda, \phi)$ are feasible for their respective problems and the primal-dual pair satisfy the following complementary slackness conditions:

$$
\begin{array}{cc}
x_{i j}>0 & \text { then } \lambda_{i j}=0 \\
x_{i j}<h_{i j} & \text { then } \phi_{i j}=0
\end{array}
$$

Let $x_{i j}^{0}$ be the given feasible solution of (1) and if we define $L=\left\{(i, j) / x_{i j}^{0}=0\right\}, F=\left\{(i, j) / 0<x_{i j}^{0}<\right.$ hij and $U=i, j / x i j O=h i j$, then the complementary slackness conditions can be restate as:

$$
\begin{array}{ll}
\lambda_{i j}=0 & \text { for all }(i, j) \notin L \\
\phi_{i j}=0 & \text { for all }(i, j) \notin U
\end{array}
$$

Now the inverse problem is to adjust the cost coefficients in such a way that the given solution $x_{i j}^{0}$ becomes optimal and the total adjustment in the cost coefficients is minimum under $l_{1}, l_{2}$, or $l_{\infty}$ norm.

Let $d$ is the adjusted value of $c$, if we replace all cost coefficients $c_{i j}$ by $d_{i j}$ in the dual constraints and apply the complementary slackness conditions (5) then we have the following characteristics of the modified cost $d_{i j}$ :

$$
\begin{array}{ll}
u_{i}+v_{j}+\lambda_{i j}=d_{i j} & \text { for all }(i, j) \in L \\
u_{i}+v_{j}-\phi_{i j}=d_{i j} & \text { for all }(i, j) \in U \\
u_{i}+v_{j}=d_{i j} & \text { for all }(i, j) \in F \\
\lambda_{i j} \geq 0, \phi_{i j} \geq 0, i=1,2, \ldots, m, j=1,2, \ldots, n .
\end{array}
$$

If we consider the $l_{1}$ norm, which is defined as $\|d-c\|=\sum\left|d_{j}-c_{j}\right|$ then the inverse problem under $l_{1}$ norm is to minimize $\sum_{i} \sum_{j}\left|d_{i j}-c_{i j}\right|$. We know that minimizing $\left|d_{i j}-c_{i j}\right|$ is equivalent to minimize $\alpha_{i j}+\beta_{i j}$, subject to the condition $d_{i j}-c_{i j}=\alpha_{i j}-\beta_{i j}$, Where $\alpha_{i j} \geq 0, \beta_{i j} \geq 0$ are respective increment and decrement in $c_{i j}$, therefore both $\alpha_{i j}$ and $\beta_{i j}$ can not take positive values. Using this transformation the inverse transportation problem can be formulated as: 
Min $\quad \sum_{i} \sum_{j}\left(\alpha_{i j}+\beta_{i j}\right)$

s.t.

$$
\begin{array}{ll}
u_{i}+v_{j}+\lambda_{i j}-\alpha_{i j}+\beta_{i j}=c_{i j} & \text { for all }(i, j) \in L \\
u_{i}+v_{j}-\phi_{i j}-\alpha_{i j}+\beta_{i j}=c_{i j} \quad \text { for all }(i, j) \in U \\
u_{i}+v_{j} \quad-\alpha_{i j}+\beta_{i j}=c_{i j} \quad \text { for all }(i, j) \in F \\
\alpha_{i j}, \beta_{i j}, \lambda_{i j}, \phi_{i j} \geq 0, i=1,2, \ldots, m, \quad j=1,2, \ldots, n .
\end{array}
$$

If we define $\bar{c}_{i j}=c_{i j}-u_{i}-v_{j}$, where $u_{i}$ and $v_{j}$ are dual variables then (7) can be written as:

Min

$$
\sum_{i} \sum_{j}\left(\alpha_{i j}+\beta_{i j}\right)
$$

s.t.

$$
\begin{array}{cc}
\lambda_{i j}-\alpha_{i j}+\beta_{i j}=\bar{c}_{i j} & \text { for all }(i, j) \in L \\
-\phi_{i j}-\alpha_{i j}+\beta_{i j}=\bar{c}_{i j} & \text { for all }(i, j) \in U \\
-\alpha_{i j}+\beta_{i j}=\bar{c}_{i j} & \text { for all }(i, j) \in F \\
\alpha_{i j}, \beta_{i j}, \lambda_{i j}, \phi_{i j} \geq 0, i=1,2, \ldots, m, j=1,2, \ldots, n .
\end{array}
$$

Where $\bar{c}_{i j}=c_{i j}-u_{i}-v_{j}, u_{i}$ and $v_{j}$ are dual variables. Now using the optimality condition of capacitated transportation problem, we can consider the following cases:

Case (1) when $\bar{c}_{i j}>0$

The non negativity of $\alpha_{i j}$ and $\beta_{i j}$ and the fact that we wish to minimize $\alpha_{i j}+\beta_{i j}$ implies that

(i) $\quad(i, j) \in L$ than $\lambda_{i j}=\bar{c}_{i j}, \alpha_{i j}=\beta_{i j}=0$ and hence $d_{i j}=c_{i j}=\left|\bar{c}_{i j}\right|$

(ii) $\quad(i, j) \in F \cup U$ than $\lambda_{i j}=\phi_{i j}=0, \beta_{i j}=\bar{c}_{i j}=\left|\bar{c}_{i j}\right|$ and hence $d_{i j}=c_{i j}-\left|\bar{c}_{i j}\right|$

Case (2) when $\bar{c}_{i j}<0$

(i) if $(i, j) \in U$ then $\phi_{i j}=-\bar{c}_{i j}=\left|\bar{c}_{i j}\right|, \alpha_{i j}=\beta_{i j}=0$ and hence $d_{i j}=c_{i j}=\left|\bar{c}_{i j}\right|$

\begin{tabular}{|c|c|c|c|c|}
\hline 12 & 13 & 5 & 20 & 25 \\
\hline 10 & 5 & 6 & 7 & \\
\hline 14 & 20 & 10 & 9 & 25 \\
\hline 8 & 2 & 7 & 6 & \\
\hline 18 & 4 & 25 & 7 & 50 \\
\hline 9 & 3 & 4 & 8 & \\
\hline 15 & 20 & 30 & 35 & \\
\hline
\end{tabular}

(ii) $(i, j) \in F \cup L$ then $\beta_{i j}=\lambda_{i j}=0, \alpha_{i j}=-\bar{c}_{i j}=\left|\bar{c}_{i j}\right|$, and hence $d_{i j}=c_{i j}+\left|\bar{c}_{i j}\right|$

Case (2) when $\bar{c}_{i j}=0$

In this case $\alpha_{i j}=\beta_{i j}=\lambda_{i j}=0$ and hence $d_{i j}=c_{i j}$.

Using above case analysis the optimal cost vector $d_{i j}^{*}$ can be given as

$d_{i j}^{*}= \begin{cases}c_{i j}-\left|\bar{c}_{i j}\right| & \text { if } \bar{c}_{i j}>0 \text { and } x_{i j}^{0}>0 \\ c_{i j}+\left|\bar{c}_{i j}\right| & \text { if } \bar{c}_{i j}<0 \text { and } x_{i j}^{0}<h_{i j} \\ c_{i j} & \text { otherwise. }\end{cases}$

\begin{tabular}{|c|c|c|c|c|c|c|c|c|}
\hline & 12 & & 13 & (5) & 5 & (20) & 20 & 25 \\
\hline 2 & 10 & 3 & 5 & 0 & 6 & 0 & 7 & $\mathrm{u}_{1}=-1$ \\
\hline & 14 & (16) & 20 & & 10 & (9) & 9 & 25 \\
\hline 0 & 8 & 0 & 2 & 1 & 7 & -1 & 6 & $\mathrm{u}_{2}=-1$ \\
\hline (15) & 18 & (4) & 4 & (25) & 25 & (6) & 7 & 50 \\
\hline 0 & 9 & 0 & 3 & -3 & 4 & 0 & 8 & $\mathrm{u}_{3}=0$ \\
\hline 15 & $v_{1}=9$ & 20 & $v_{2}=3$ & 30 & $v_{3}=7$ & 35 & $v_{4}=8$ & $\mathrm{z}^{*}=551$ \\
\hline
\end{tabular}

Thus we can solve the inverse problem by solving equation (7) and optimal cost vector $d_{i j}^{*}$ can be obtained by (9), but instead of solving (7) we can calculate the optimal dual variables by using two phase method and the modified costs can be calculated with the help of (9).

\section{Numerical Example}

Let us consider a capacitated transportation problem

In the above rectangular array; the numbers written in the top right corner of each cell represents the upper bound of each variable, the numbers at the bottom right corner represent the unit transportation cost and the numbers written in the last column and last raw represent the available quantities and requirements at each source and destination respectively.

Solving the problem using two phase method we obtain the final transportation table as follows: 
Where $\mathrm{u}_{1}=-1, \mathrm{u}_{2}=-1, \mathrm{u}_{3}=0, \mathrm{v}_{1}=9, \mathrm{v}_{2}=3, \mathrm{v}_{3}=7, \mathrm{v}_{4}=8$ are the optimal dual variables, $x^{*}=\left\{x_{13}=5, x_{14}=20\right.$, $x 22=16, x 24=9, x 31=15, x 32=4, x 33=25, x 34=6$ is the optimal solution and $\mathrm{z}^{*}=551$ is the minimum transportation cost. The number written on the bottom left corner of each cell in the last table is $\bar{c}_{i j}$.

Now let us assume that $x^{0}=\left\{x_{13}=5, x_{14}=20, x_{22}=17, x_{24}=8, x_{31}=15, x_{32}=3, x_{33}=25, x_{34}=\right.$ 7 is the given feasible solution. Using (9) the modified costs can be calculated as

$d_{24}^{*}=c_{24}+\left|\bar{c}_{24}\right|=c_{24}+\left|c_{24}-u_{2}-v_{4}\right|=6+1=7$

We can easily verify that $x^{0}$ is an optimal solution of the modified transportation problem.

\section{Conclusion}

Inverse optimization is an important area in both academic research and practical applications. Using the inverse optimization this paper suggested an inverse based methodology for the solution of capacitated transportation problem. An illustration observation used to demonstrate the advantage of the new approach. For the future work this approach can be used on transportation problem with linear fractional objective function

\section{References}

[1] Burton, D., Toint, Ph. L. (1992). On an instance of shortest paths problem. Mathematical Programming, 53, 45-61.

[2] Zhang, J., \& Liu, Z. (1996). Calculating some inverse linear programming problems. Journal of Computational and Applied Mathematics. 72, 261-273.

[3] Zhang, J., \& Liu, Z. (1999). A further study on inverse linear programming problems. Journal of Computational and Applied Mathematics. 106, 345-359.

[4] Ahuja, R. K. \& Orlin, J. B. (2001). Inverse Optimization. Operation Research, 49,771-783.

[5] Huang, S. \& Liu, Z. (1999). On the inverse problem of linear programming and its application to minimum weight perfect kmatching. European Journal of Operational Research, 112, 421-426.

[6] Amin, G.R., Emrouznejad, A. (2007). Inverse Forecasting: A New approach for predictive modeling, Computers \& Industrial Engineering. DOI:10.1016/j.cie. 2007.05.007.

[7] Yibing, L., Tiesong, H., Zhongping, W. (2008). A penalty function method for solving inverse optimal value problem. J. Comp Appl. Math., 220(1-2):175-180.

[8] Zhang, J., Zhang, Li., Xiao, X. (2010). A perturbation approach for an inverse quadratic programming problem. doi: 10.1007/s00186010-0323-4.

[9] Zhang, J., Zhang, Li. (2010). An Augmented Lagrangian Method for a Class of Inverse Quadratic Programming Problems. Applied Math. \& Opt. 61(1), 57-83.

[10] Zhang, J., Zhang, Li. (2009). Solving a class of inverse QP problems by a smoothing Newton method. Journal of Computational Mathematics, 27:6, 787-801.

[11] Wang, L. (2009). Cutting plane algorithms for the inverse mixed integer linear programming problem. Operations Research Letters 37, 114-116.

[12] Hladik, M. (2010). Generalized linear fractional programming under interval uncertainty. Eur. J. Oper. Res., 205(1), 42-46.

[13] Jaing, Y., Xiao, X., Zhang, Li. and Zhang, J. (2011). A perturbation approach for a type of inverse linear programming problem. DOI: $10.1080 / 00207160903513003$.

[14] Jain, S. and Arya, N. (2012). Inverse linear fractional Programming: A new approach. Journal of Computer and Mathematical Sciences. Vol. 3(5), 542-547.

[15] Hitchcock, Frank, L. (1941). The Distribution of a Product from Several Sources tonumerous localities. J. Math. Phys. 20, $224-230$.

[16] Kantorivich, L. V. (1942). On the Translocation of Masses, Compt. Rend. Acad. Sci.U.S.S.R. 37, 199-201.

[17] Kantorivich, L.V. and Gavurin, M.K. (1949). The Application of Mathematical Methods to Freight Flow Analysis, (translation), Akademii Nauk SSSR.

[18] Dantzig, G. B. and Thapa, M. N. (1997). Linear Programming1: Introduction. Springer

[19] Jain, S. (2009). A dual matrix approach for unbalanced transportation problem. Int. Trans. in Mathematical Sciences \& Computer, 2(1), 61-71. 